\title{
Use of the Prostate Health Index and Density in 3 Outpatient Centers to Avoid Unnecessary Prostate Biopsies
}

\author{
Anni Schulze $^{a}$ Frank Christoph $^{b}$ Markus Sachs $^{c} \quad$ Joerg Schroeder $^{d}$ \\ Carsten Stephan $^{e}$ Martin Schostak $^{f}$ Frank Koenigd, $^{d, f}$ \\ ${ }^{a}$ Department of Surgery, Helios Klinikum Jerichower Land, Burg, Germany; ${ }^{b}$ Urological Practice Urologie Citywest,

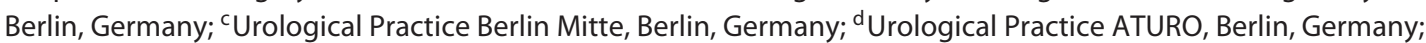 \\ eDepartment of Urology, Charité-Universitätsmedizin Berlin, Corporate Member of Freie Universität Berlin and \\ Berlin Institute for Urologic Research, Berlin, Germany; ${ }^{\mathrm{f} D e p a r t m e n t}$ of Urology, Uro-Oncology, Robot-Assisted and \\ Focal Therapy, University Clinic Magdeburg, Magdeburg, Germany
}

\section{Keywords}

Prostate health index · Prostate health index density · PSA · Prostate cancer · Prostate biopsy

\footnotetext{
Abstract

Objectives: We investigated the diagnostic efficacy of the prostate health index (PHI) and PHI density (PHID) to avoid unnecessary prostate biopsies in 3 urological practices. Methods: In 122 patients, total prostate-specific antigen (PSA), free PSA (f-PSA), the quotient from total PSA and $f-P S A$ (f-PSA\%), and [-2]pro-PSA were measured in the serum; $\mathrm{PHI}$, PHID, and PSA density (PSAD) were calculated prior to prostate biopsy. Tissue sampling via transrectal biopsy was indicated in case of suspicious PSA (progression and/or elevation of PSA) and/or suspicious digital rectal examination. PSAD, PHI, and PHID were not used for biopsy indication. The diagnostic efficacy was determined with receiver-operating characteristic (ROC) and decision curve analyses. Results:
}

Based on prostate biopsies, 38\% $(n=46)$ of the cases had no prostate carcinoma (PCa), $21 \%(n=26)$ no clinically significant (insignificant) PCa, and $41 \%(n=50)$ had clinically significant PCa. ROC analyses of the PSA parameters showed higher diagnostic efficacy for PHI and PHID (AUC 0.722 and 0.739 ) than for f-PSA\%, PSA, and PSAD (AUC 0.612, 0.595, and 0.698 , respectively) regarding carcinoma diagnosis. With a combined use of PHI and PHID (cutoff $>40$ and $>0.9$, respectively), only 1 clinically significant PCa would have been missed (sensitivity 98\%); in 24 (20\%) patients, biopsy could have been avoided. Conclusion: The integration of $\mathrm{PHI}$ and PHID could improve the diagnostic efficacy of risk calculators to avoid unnecessary prostate biopsies. However, as a prerequisite, validation of cutoff values in prospective studies is urgently required.

(c) 2020 S. Karger AG, Basel

Anni Schulze and Frank Christoph contributed equally to this work. Martin Schostak and Frank Koenig shared last authorship. 


\section{Introduction}

According to the current S3 guideline in Germany, a prostate biopsy should be conducted at a prostate-specific antigen (PSA) elevation of $>4 \mathrm{ng} / \mathrm{mL}$ (after conduction of a control measurement and considering influencing factors) in the early detection of prostate carcinoma (PCa) [1].

The results of the ERSPC (European Randomized Study of Screening for Prostate Cancer) showed that of 16,600 screened patients, a prostate biopsy is conducted in 3,104 patients, and only in approximately $25 \%$ of these patients a carcinoma is found [2].

That means that in $75 \%$ of the cases, an invasive procedure with potential risks such as bleeding, infection, intestinal injury, and pain is conducted unnecessarily in unaffected men. Searching for a way out of this diagnostic dilemma, based on recent study data on questionable PSA elevation, German and European guidelines recommend the additional use of multiparametric magnetic resonance imaging (mpMRI) of the prostate and the use of validated nomograms prior to a planned prostate biopsy. The European Organization for Research and Treatment of Cancer (EORTC) recommends the risk calculator (RC) of the Prostate Cancer Research Foundation (SWOP) as such a nomogram [3]. Besides PSA, prostate volume, digital rectal examination (DRE), and age, the RC can be optimized by including mpMRI results and the prostate health index (PHI). For several years now, PHI has been recommended as an additional marker in the early diagnosis of $\mathrm{PCa}$, especially in case of PSA elevations between 2 and $10 \mathrm{ng} / \mathrm{mL}$. In addition to total PSA (t-PSA) and free PSA (f-PSA), PHI also includes [-2]pro-PSA, which results in a significantly higher diagnostic efficacy than the individual parameters $[4,5]$. Based on retrospective data analysis, the present work examines the benefit of PHI and the recently suggested PHI density (PHID = PHI divided by the prostate volume) [6] in the daily routine of urological practices.

\section{Materials and Methods}

Between 2012 and 2018, prostate biopsies were conducted in 122 consecutive patients in 3 urological practices in Berlin based on a suspicious PSA (progression and/or elevation of PSA) and/or suspicious DRE.

The results of DRE were abnormal in $12 \%$ of the patients. Prior to the planned prostate biopsy, t-PSA, f-PSA, and [-2]pro-PSA were measured in serum followed by the calculation of PHI, PHID, and PSA density (PSAD) for all patients. PSA values were measured with the standard laboratory device UniCel DxI 600 access immunoassay system (Beckman Coulter, Hybritech method). Since [-2]pro-PSA and f-PSA is relatively unstable in com-
Table 1. Age, prostate volume, PSA, PSAD, PHI, PHID, and f-PSA\% of the study patients $(n=122)$

\begin{tabular}{|c|c|c|c|c|}
\hline Parameter & Median & Mean & Min & $\operatorname{Max}$ \\
\hline Age, years & 65 & 65.41 & 41 & 81 \\
\hline Prostate volume, $\mathrm{cm}^{3}$ & 37 & 46.66 & 14 & 190 \\
\hline $\mathrm{PSA}, \mathrm{ng} / \mathrm{mL}$ & 6.81 & 9.55 & 1.91 & 82.9 \\
\hline PSAD & 0.18 & 0.25 & 0.02 & 2.3 \\
\hline PHI & 59.25 & 71.87 & 12.60 & 312 \\
\hline PHID & 1.59 & 2.05 & 0.094 & 8.67 \\
\hline f-PSA $\%$ & 11 & 12.35 & 3 & 43 \\
\hline
\end{tabular}

parison to t-PSA, all blood samples collected were centrifuged about 10 min after clotting and separated into serum at least within $1 \mathrm{~h}$ after blood sampling. Serum was kept at $4^{\circ} \mathrm{C}$ until processed for analysis, which usually took place immediately or at least within $24 \mathrm{~h}$. All measurements were performed on one machine.

The median t-PSA value was $6.81 \mathrm{ng} / \mathrm{mL}(1.91-82.9 \mathrm{ng} / \mathrm{mL})$. The median age of the patients was 65 years ( $41-81$ years). The median prostate volume was $37 \mathrm{~cm}^{3}\left(14-190 \mathrm{~cm}^{3}\right)$. PHI was calculated as follows:

$$
\mathrm{PHI}=[-2] \text { pro-PSA } / \mathrm{f}-\mathrm{PSA} \times \sqrt{ } \mathrm{t}-\mathrm{PSA} .
$$

Analogous to the calculation of PSAD, PHID was calculated as follows:

$$
\mathrm{PHID}=\mathrm{PHI} / \text { prostate volume }
$$

The correlation of serum markers with pathological findings was determined using Spearman rank correlation (Spearman's $\rho$ ) and the significance test as in Meng et al. [7]. Moreover, receiver-operating characteristic (ROC) curve analyses were imputed. In addition, decision curve analysis was performed as described earlier [8]. Decision curve analysis identifies the range of threshold probabilities and their benefit, i.e., where the model or biomarker is of value [9].

Biopsies were collected as outpatient procedure using antibiosis and local anesthesia. The procedure was supplemented by transrectal ultrasound. Depending on the prostate size, 10-14 tissue samples were collected per patient. Histological examination was performed at the pathological institute associated with the respective practice.

\section{Results}

In 46 prostate biopsies (38\%), no evidence for PCa was found. In $26(21 \%)$ patients, clinically insignificant (Gleason $=3+3)$ PCa was diagnosed. In $50(41 \%)$ of the 122 biopsied patients, clinically significant (Gleason $>3+3$ ) PCa was detected.

Results of t-PSA, f-PSA\%, PSAD, PHI, and PHID measurements, calculations (median, mean, min, and max), and descriptive data (age and prostate volume) of the co- 
Table 2. PSA, PSAD, PHI, PHID, and f-PSA\% in the group with no PCa $(n=46)$

\begin{tabular}{lrrrr}
\hline Parameter & Median & Mean & \multicolumn{1}{l}{ Min } & \multicolumn{1}{c}{ Max } \\
& & & & \\
\hline PSA, ng/mL & 6.1 & 7.03 & 1.91 & 17.06 \\
PSAD & 0.13 & 0.16 & 0.04 & 0.16 \\
PHI & 43.60 & 53.34 & 12.60 & 150.10 \\
PHID & 1.05 & 1.34 & 0.094 & 5.20 \\
f-PSA\% & 13 & 13.87 & 3 & 43 \\
\hline
\end{tabular}

Table 4. PSA, PSAD, PHI, PHID, and f-PSA\% in the group with low-risk, clinically insignificant PCa (GS $=3+3 ; n=26)$

\begin{tabular}{lrrrr}
\hline Parameter & Median & Mean & \multicolumn{1}{c}{ Min } & \multicolumn{1}{c}{ Max } \\
\hline PSA, ng/mL & 6.03 & 7.45 & 2.42 & 28.52 \\
PSAD & 0.16 & 0.19 & 0.02 & 0.66 \\
PHI & 58.65 & 60.09 & 19.80 & 130.00 \\
PHID & 1.51 & 1.81 & 0.19 & 5.2 \\
f-PSA\% & 11.5 & 13.05 & 6.2 & 31 \\
\hline
\end{tabular}

hort are presented in Table 1. Results of t-PSA, f-PSA\%, PSAD, PHI, and PHID measurements and calculations (median, mean, min, and max) for men without PCa and PCa patients (total) are presented in Tables 2 and 3; the results, separated in clinically insignificant (Gleason = $3+3$ ) and clinically significant (Gleason $>3+3$ ) PCa, are presented in Tables 4 and 5 .

Based on these measurements, the diagnostic efficacy of the individual parameters was assessed by ROC analysis. The results of all PCa diagnosed (Fig. 1) point to a higher efficacy for PHI and PHID (AUC 0.722 and 0.739, respectively) than f-PSA\%, PSA, and PSAD (AUC 0.612, 0.595 , and 0.698 , respectively). The difference between PHI and PHID in comparison to PSA was statistically significant with $p$ values of 0.019 and 0.025 , respectively. However, PSAD was also significantly better than PSA $(p=0.014)$. The differences in AUC between PHI and PHID, as well as the differences between PHI and PHID in comparison to PSAD, were not statistically significant $(p>0.05)$.

Focusing on the diagnosis of clinically significant carcinomas only, the results of ROC analysis were better for PHI and PHID (AUC 0.757 and 0.764, respectively) than for f-PSA\%, PSA, and PSAD (AUC 0.627, 0.635, and 0.732 , respectively), too (Fig. 2). With $p$ values for PHI and PHID of 0.022 and 0.041 , respectively, these differ-
Table 3. PSA, PSAD, PHI, PHID, and f-PSA\% in the group with $\mathrm{PCa}$ (total) $(n=76)$

\begin{tabular}{lrrrr}
\hline Parameter & Median & Mean & \multicolumn{1}{c}{ Min } & \multicolumn{1}{c}{ Max } \\
& & & & \\
\hline PSA, ng/mL & 7.56 & 11.07 & 2.23 & 82.90 \\
PSAD & 0.19 & 0.30 & 0.02 & 2.30 \\
PHI & 65.75 & 83.09 & 19.80 & 312.00 \\
PHID & 2.015 & 2.47 & 0.19 & 8.67 \\
f-PSA\% & 10 & 11.49 & 3 & 31 \\
\hline
\end{tabular}

Table 5. PSA, PSAD, PHI, PHID, and f-PSA $\%$ in the group with clinically significant PCa $(\mathrm{GS}>3+3 ; n=50)$

\begin{tabular}{lrrrr}
\hline Parameter & Median & Mean & \multicolumn{1}{c}{ Min } & \multicolumn{1}{c}{ Max } \\
\hline PSA, ng/mL & 8.03 & 12.95 & 2.23 & 82.90 \\
PSAD & 0.22 & 0.36 & 0.07 & 2.30 \\
PHI & 77.20 & 95.05 & 28.60 & 312.00 \\
PHID & 2.27 & 2.82 & 0.56 & 8.67 \\
f-PSA\% & 10 & 10.66 & 3 & 23.4 \\
\hline
\end{tabular}

ences in PSA were again statistically significant. As for the analysis of all carcinomas, PSAD was significantly better than PSA $(p=0.011)$. In contrast, the differences between PHI and PHID as well as the differences between PHI and PHID in comparison to PSAD were not statistically significant $(p>0.05)$. In addition to the results described above, decision curve analysis confirmed the superiority of PHI and PHID over t-PSA in PCa diagnosis (Fig. 3, 4).

With a combined application of PHI and PHID and cutoff values of $>40$ and $>0.9$, respectively, biopsies could have been avoided in 24 (20\%) patients. Only one Gleason $4+4$ carcinoma would have been missed (sensitivity $98 \%)$. In this patient, the tumor had a small portion of the volume within an enlarged prostate $\left(51 \mathrm{~cm}^{3}\right)$. Only 2 of 12 biopsies were positive. This was confirmed by final histology following radical prostatectomy. Besides a downgrading to Gleason $4+3$, evidence for the tumor was only found in 1 lobe (pT2a).

\section{Discussion}

Despite all limitations, PSA is still the most important biomarker for the early diagnosis of PCa. However, the uncritical use of this biomarker results in overdiagnosis 


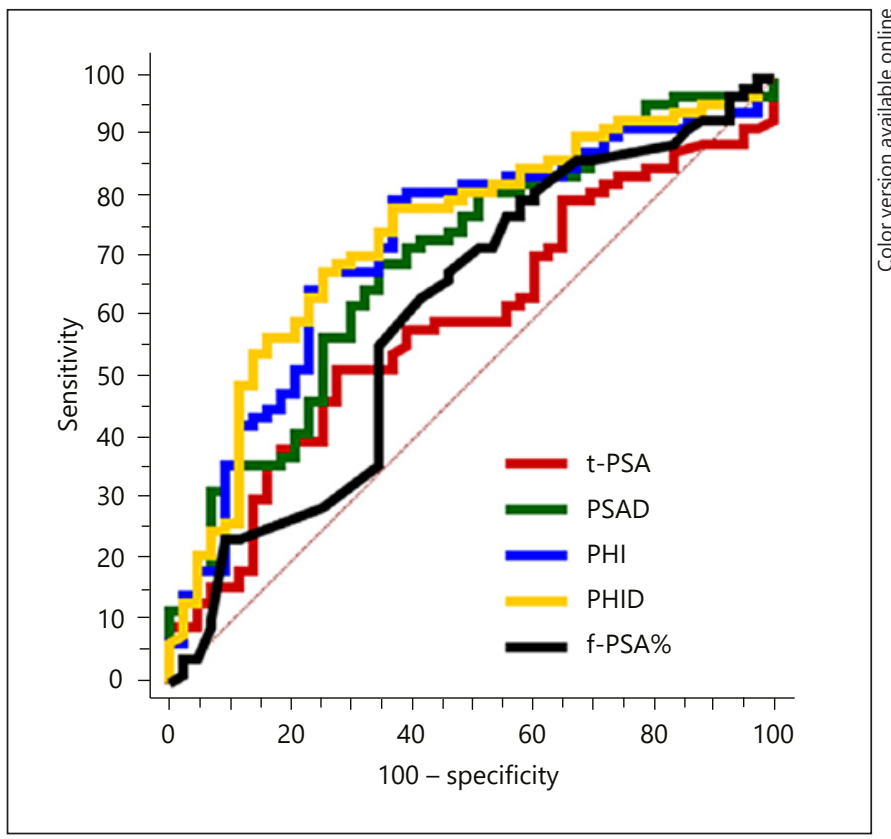

Fig. 1. Receiver-operating characteristic curve of PSA, PSAD, PHI, PHID, and f-PSA\% in the group of men with PCa (total).

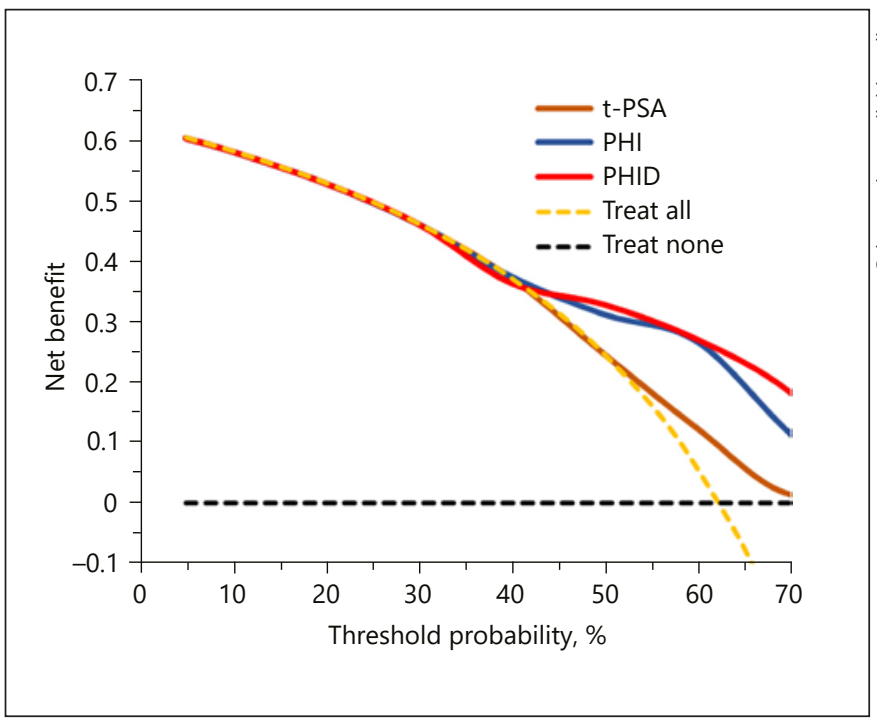

Fig. 3. Decision curve analysis of t-PSA, PHI, and PHID in the group of men with PCa (total).

and consequently in overtreatment. Thus, global guidelines recommend risk- and age-adapted PSA measurements instead of a general screening. A notable improvement in the diagnostic efficacy can be achieved by the additional assessment of PSA isoforms. PHI combines

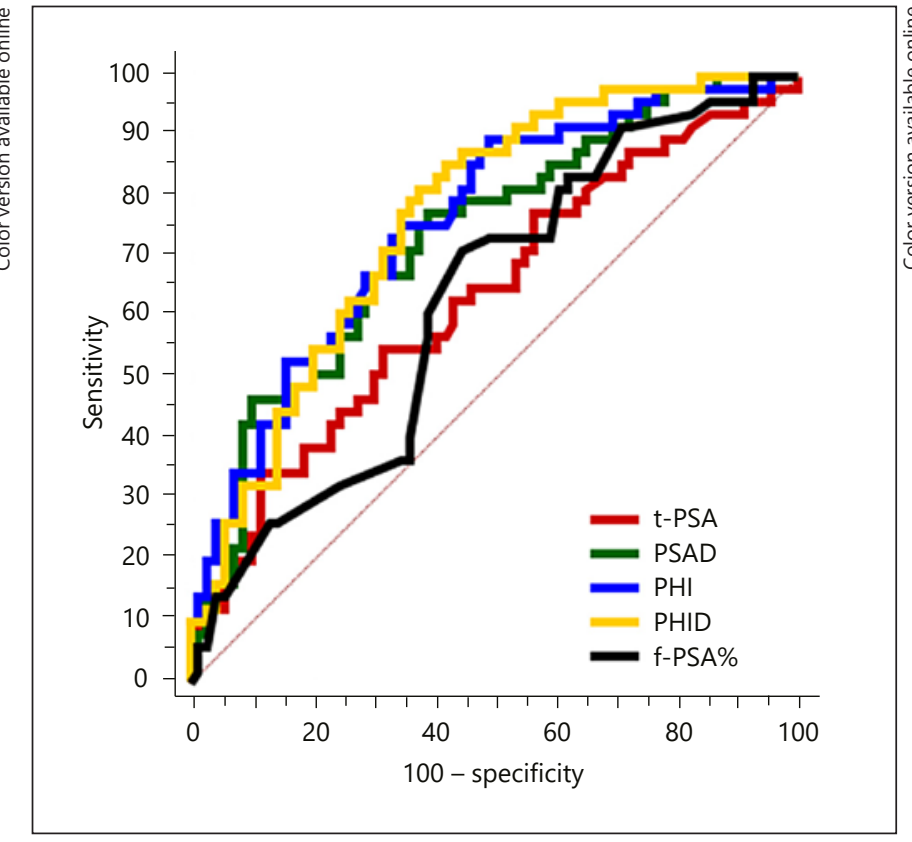

Fig. 2. Receiver-operating characteristic curve of PSA, PSAD, PHI, PHID, and $\mathrm{f}-\mathrm{PSA} \%$ in the group of men with clinically significant PCa.

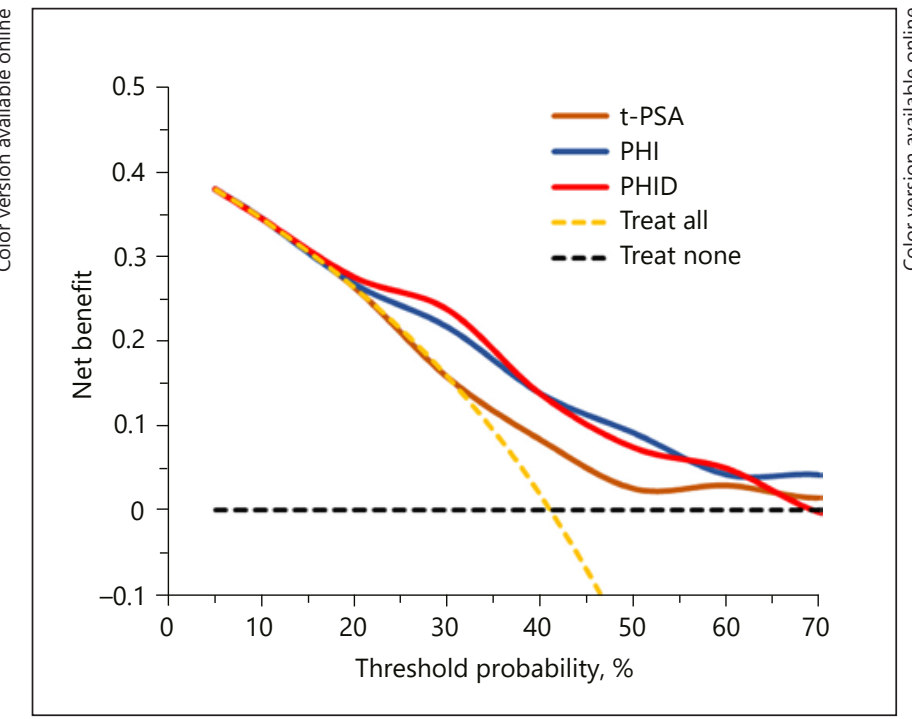

Fig. 4. Decision curve analysis of t-PSA, PHI, and PHID in the group of men with clinically significant $\mathrm{PCa}$.

the results of 3 quantitative kallikrein immunoassays (t-PSA, f-PSA, and [-2]pro-PSA) with the calculation of 1 numeric score. Based on solid data, PHI was approved by the Food and Drug Administration (FDA) already in 2012 for the differentiation of benign and malignant 
prostate changes in men $>50$ years of age with a PSA value of $4-10 \mathrm{ng} / \mathrm{mL}$ and normal DRE findings [10]. Based on numerous studies, the manufacturer (Beckman Coulter) provides carcinoma probabilities depending on the PSA value: a probability of $8.4 \%$ for values $<21 ; 21 \%$ for values $<40$, and $44 \%$ for values $\geq 40$.

However, the use of cutoff values is more practical in daily routine. Recently, a multicenter study with $800 \mathrm{Eu}-$ ropean patients established a sensitivity of $90 \%$ for the diagnosis of clinically significant carcinomas. Clinical significance was defined as Gleason $\geq 3+3$ with a PHI $>40$. Using this cutoff, $40 \%$ of all biopsies could have been prevented as well as the diagnosis of a Gleason $3+3$ tumor in $31 \%$ of the cases [11]. Interestingly, with the same sensitivity, a cutoff value for PHI of $30 \%$ was determined in the same study for 1,688 Asian men. Related to this result was a possible avoidance of a biopsy and diagnosis of Gleason $3+3$ tumors for $56 \%$ and $33 \%$ of the men analyzed, respectively. Obviously, ethnic differences exist between both study populations.

In recent years, it was shown that PHID reaches higher AUC values in ROC analyses in comparison to PHI. Thus, PHID allows for an improved prediction of clinically significant PCa prior to a prostate biopsy. However, available data to support this finding are limited. PHID was first described in 2014 by Mearini et al. [6]. In their study, they analyzed PHID in 275 patients with a notably higher AUC of 0.77 compared to t-PSA (AUC $=0.54$ ). However, the advantage in comparison to PHI with an AUC of 0.76 was only marginal.

Since then, only 2 more studies on PHID were published $[12,13]$. In a study in 118 patients in 2017, Tosoian et al. [13] determined thus far the highest value for PHID in the ROC analysis with an AUC of 0.84 and a PHID median of 1.21 for clinically significant PCa. For PHI and t-PSA, AUCs of 0.76 and 0.52, respectively, were determined. Based on these values, a cutoff value of 0.43 for PHID was suggested for clinically significant carcinomas (Gleason $>3+3$ ) with a sensitivity of $100 \%$. In our own study, we could also demonstrate an improved diagnostic efficacy for PHID. However, AUC was only 0.76 , and we determined a cutoff value of 0.9 to achieve a similarly high sensitivity of $98 \%$. In addition, we calculated a higher AUC of 0.64 for t-PSA. Our median PHID value for clinically significant carcinomas was notably higher (2.27), too. A possible explanation for these different values might be the different disposition of study cohorts. In our study, only $38 \%$ of the men did not have a carcinoma in comparison to $60 \%$ in the study by Tosoian et al. The portion of clinically sig- nificant carcinomas was also higher in our cohort (41 vs. $30 \%)$.

To determine a reliable cutoff value for PHID that is useful in daily practice, considerably higher patient numbers need to be studied in controlled prospective clinical trials. In these trials, tumor volume as well as the type of biopsy should be considered besides the Gleason score, because the detection rate of prostate carcinomas is notably improved by the increasing application of MRI fusion biopsies. Recently, Lopes Vendrami et al. [14] described a possible influence of the biopsy type on the diagnostic efficacy of PHI and PHID prior to a biopsy. In their study, PHI and PHID was determined prior to prostate biopsy in 211 men. Subsequently, MRI fusion prostate biopsy was conducted in 90 cases and a standard prostate biopsy in 121 cases. For PCa prediction following fusion biopsy, the highest AUC was found for PHID with 0.82 . In contrast, for the standard biopsy, PHI was found to have the better AUC (0.78). With cutoff values of 0.64 for PHID in combination with fusion biopsies and values of 45.9 for PHI with standard biopsies, the rate of tumor-negative biopsies could have been reduced by 63.4 and $77.3 \%$, respectively. However, this benefit would have been achieved at the cost of missing clinically significant tumors at a rate of 12 and 19\%, respectively.

When discussing the topic of PCa screening, considering the cost factor for the health care system is crucial. In the last years, the application and standardization of mpMRI was shown to lead to a notable improvement in early PCa diagnosis. A combination of mpMRI and PHID for the detection of clinically significant carcinomas with a concomitant reduction in unnecessary biopsies is plausible and was described by Druskin et al. [15] in 2018, who conducted a study in 241 patients. For reasons of cost efficiency, however, the application of PHID (cost: approx. EUR 100) to select suitable patients prior to mpMRI (cost: several hundreds of euros) will be of great interest in the future. In this context, in 2019, Osses et al. [16] suggest a triage prior to mpMRI in their current review. This means that in patients with clinical suspicion of $\mathrm{PCa}$ (suspicious progression and/or elevated t-PSA and/or abnormal DRE findings), RC could be applied first. RC by SWOP was used and validated in the ERSPC study [3]. In this RC, t-PSA, age, DRE, and transrectal ultrasound were initially integrated. More recently, the calculator was expanded by PHI. Based on the data from previous studies and our own current investigation, we think that PHID might be the better parameter to estimate the PCa risk. 


\section{Conclusion}

Using PHI and PHID, unnecessary biopsies could be avoided, and the diagnosis of clinically insignificant tumors (active surveillance population) could be reduced. Prior to their application in daily routine, the validation of cutoff values in prospective studies is indispensable. The cutoff values need to be established before a general recommendation for the $\mathrm{PHI}$ measurement can be given and before an integration of PHI/PHID into multiparametric RCs is possible.

\section{Statement of Ethics}

The published article complies with the guidelines for human studies and was conducted ethically in accordance with the World Medical Association Declaration of Helsinki. Since it was a retrospective analysis of pseudo-anonymized data, no ethical approval was required.

\section{Disclosure Statement}

None of the authors has any financial interests (stocks, patents, employment, honoraria, or royalties) or nonfinancial relationship (political, personal, or professional) that may be interpreted as having influenced the content of the paper.

\section{Funding Sources}

The study did not receive any funding.

\section{Author Contributions}

All authors have made substantial contributions to the conception or design of the work; or the acquisition, analysis, or interpretation of data for the work; all drafted the work or revised it critically for important intellectual content and gave their final approval of the version to be published; and agreed to be accountable for all aspects of the work in ensuring that questions related to the accuracy or integrity of any part of the work are appropriately investigated and resolved.

\section{References}

1 Interdisziplinäre Leitlinie der Qualität S3 zur Früherkennung. Diagnose und Therapie der verschiedenen Stadien des Prostatakarzinoms; Kurzversion 5.0 - April 2018; AWMFRegister-Nummer 043/022OL. Available from: https://www.leitlinienprogrammonkologie.de/fileadmin/user_upload/ Downloads/Leitlinien/Prostata 5 0/LL Prostata_Kurzversion_5.0.pdf.

2 Bokhorst LP, Zhu X, Bul M, Bangma CH, Schröder FH, Roobol MJ. Positive predictive value of prostate biopsy indicated by prostate-specific-antigen-based prostate cancer screening: trends over time in a European randomized trial. BJU Int. 2012 Dec;110(11): 1654-60.

3 http://www.prostatecancer-riskcalculator. $\mathrm{com} /$ seven-prostate-cancer-risk-calculators.

4 Loeb S, Catalona WJ. The Prostate Health Index: a new test for the detection of prostate cancer. Ther Adv Urol. 2014 Apr;6(2):74-7.

5 Boegemann M, Stephan C, Cammann H, Vincendeau S, Houlgatte A, Jung K, et al. The percentage of prostate-specific antigen (PSA) isoform [-2]proPSA and the prostate health index improve the diagnostic accuracy for clinically relevant prostate cancer at initial and repeat biopsy compared with total PSA and percentage free PSA in men aged $\leq 65$ years. BJU Int. 2016 Jan;117(1):72-9.
6 Mearini L, Ferri C, Lazzeri M, Bini V, Nunzi E, Fiorini D, et al. Evaluation of prostate-specific antigen isoform p2PSA and its derivates, $\%$ p2PSA, prostate health index and prostate dimension-adjusted related index in the detection of prostate cancer at first biopsy: an exploratory, prospective study. Urol Int. 2014;93(2):135-45

7 Meng X-L, Rubin DB. Performing likelihood ratio tests with multiply-imputed data sets. Biometrika. 1992 March;79(1):103-11.

8 Stephan C, Jung K, Semjonow A, SchulzeForster K, Cammann $\mathrm{H}$, Hu X, et al. Comparative assessment of urinary prostate cancer antigen 3 and TMPRSS2:ERG gene fusion with the serum [-2]proprostate-specific antigen-based prostate health index for detection of prostate cancer. Clin Chem. 2013 Jan;59(1): $280-8$.

9 Vickers AJ, Elkin EB. Decision curve analysis: a novel method for evaluating prediction models. BMC Med Inform Decis Making. 2006 Nov-Dec;26(6):565-74.

10 Summary of Safety and Effectiveness Data (SSED). PMA P090026. Available from: accessdata.fda.gov/cdrh_docs/pdf9/P090026b. pdf.

11 Chiu PK, Ng CF, Semjonow A, Zhu Y, Vincendeau S, Houlgatte A, et al. A Multicentre Evaluation of the Role of the Prostate Health Index (PHI) in Regions with Differing Prevalence of Prostate Cancer: Adjustment of PHI Reference Ranges is Needed for European and Asian Settings. Eur Urol. 2019 Apr;75(4): 558-61.
12 Friedl A, Stangl K, Bauer W, Kivaranovic D, Schneeweiss J, Susani M, et al. Prostate-specific antigen parameters and prostate health index enhance prostate cancer prediction with the in-bore 3-T magnetic resonance imaging-guided transrectal targeted prostate biopsy after negative 12-core biopsy. Urology. 2017 Dec;110:148-53.

13 Tosoian JJ, Druskin SC, Andreas D, Mullane P, Chappidi M, Joo S, et al. Prostate health index density improves detection of clinically significant prostate cancer. BJU Int. 2017 Dec; 120(6):793-8.

14 Lopes Vendrami C, McCarthy RJ, Chatterjee A, Casalino D, Schaeffer EM, Catalona WJ, et al. The utility of prostate specific antigen density, prostate health index, and prostate health index density in predicting positive prostate biopsy outcome is dependent on the prostate biopsy methods. Urology. 2019 Jul;129:153-9.

15 Druskin SC, Tosoian JJ, Young A, Collica S, Srivastava A, Ghabili K, et al. Combining prostate health index density, magnetic resonance imaging and prior negative biopsy status to improve the detection of clinically significant prostate cancer. BJU Int. 2018 Apr; 121(4):619-26.

16 Osses DF, Roobol MJ, Schoots IG. Prediction medicine: biomarkers, risk calculators and magnetic resonance imaging as risk stratification tools in prostate cancer diagnosis. Int J Mol Sci. 2019 Apr;20(7):E1637. 